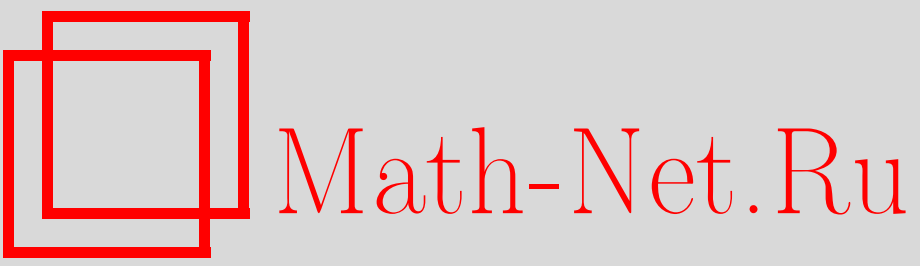

И. Д. Шкредов, О некоторых аддитивных задачах, связанных с показательной функцией, УМН, 2003, том 58, выпуск 4, 165-166

DOI: https://doi.org/10.4213/rm657

Использование Общероссийского математического портала Math-Net.Ru подразумевает, что вы прочитали и согласны с пользовательским соглашением

http://www.mathnet.ru/rus/agreement

Параметры загрузки:

IP: 54.164 .48 .24

26 апреля 2023 г., 09:05:19 


\title{
О НЕКОТОРЫХ АДДИТИВНЫХ ЗАДАЧАХ, СВЯЗАННЫХ С ПОКАЗАТЕЛЬНОЙ ФУНКЦИЕЙ
}

\author{
И. Д. ШКРЕДОВ
}

1. Постановка задач и формулировка результатов. Для простого числа $p$ обозначим через $Z_{p}$ кольц вычетов по модулю $p$, а через $Z_{p}^{*}$ - группу обратимых элементов $Z_{p}$. Пусть $R$ - подгруппа $Z_{p}^{*}$. В работе [1] рассматривался вопрос о представимости произвольного элемента $Z_{p}^{*}$ в виде суммы элементов из $R$ и был получен следующий результат.

Теорема А. Пусть $R$ - подгруппа $Z_{p}^{*}$. Если для некоторого натурального $l \geqslant 2$ виполнено $|R|>p^{1 / 2+1 /(2 l)}$, то для произвольного $b \in Z_{p}^{*}$ существуют $x_{1}, \ldots, x_{l} \in R$ такие, что $b \equiv x_{1}+\cdots+x_{l}(\bmod p)$.

В работах [2] и [3] изучалась другая аддитивная задача. Пусть $g$ - первообразный корень по модулю $p, l \geqslant 2$ - натуральное. Рассмотрим множество разностей

$$
A^{*}:=\left\{\left(g^{n_{1}}-g^{n_{2}}, g^{n_{2}}-g^{n_{3}}, \ldots, g^{n_{l-1}}-g^{n_{l}}\right)(\bmod p): 1 \leqslant n_{1}, \ldots, n_{l} \leqslant N\right\} .
$$

В [2] была доказана следующая теорема.

ТЕОремА Б. Пусть $а$ - произвольный вектор, $a \in Z_{p}^{l-1}$, такой, что все суммы $b_{j}:=$ $\sum_{k=j}^{l-1} a_{k}, 1 \leqslant j \leqslant l-1, b_{l}:=0$ различны по модулю $p$. Пусть также $N \gg p^{1-1 /(2 l)+\varepsilon}$. Тогда а принадлежит $A^{*}$.

В работе [3] был рассмотрен случай $l=2$ и исследован вопрос о принадлежности "почти всех" вычетов из $Z_{p}$ множеству $A^{*}$.

Теорема В. Для произвольного простого $p$, первообразного корня $g \bmod p$ и любого натурального $N<p$

$$
\#\left\{h(\bmod p): h \neq g^{x}-g^{y}, 1 \leqslant x, y \leqslant N\right\} \ll \frac{p^{3} \log p}{N^{3}} .
$$

В настоящей заметке будут доказаны утверждения о разрешимости сравнений, возникающих в данных аддитивных задачах, для "почти всех" вычетов. Также будет доказано неболшшое усиление теоремы В. Доказательство теорем основано на одном методе Фреймана из [4].

ТеОрема 1. Для любого натурального $l \geqslant 2$ и любого $\varepsilon>0$ существует $C_{\varepsilon}>0$ такое, что для любой подгруппь $R \subseteq Z_{p}^{*}$ мощности $|R| \geqslant C_{\varepsilon} p^{l /(2 l-1)}$ количество $x \in Z_{p}^{*}, x \equiv x_{1}+\cdots+x_{l}(\bmod p), x_{1}, \ldots, x_{l} \in R$, больие, чем $(1-\varepsilon) p$.

Теорема 2. Для любого натурального $l \geqslant 2$ и любого $\varepsilon>0$ существует $C_{\varepsilon}>0$ такое, что для $N \geqslant C_{\varepsilon} p^{l /(l+1)}$ выполнено $\left|A^{*}\right|>(1-\varepsilon) p^{l-1}$.

ТЕОРемА 3. Для любого натурального $l \geqslant 2$, любого $\varepsilon>0$ и любого набора $b_{1}, \ldots, b_{l} \in$ $Z_{p}^{*}$ существует $C_{\varepsilon}>0$ такое, что для $N \geqslant C_{\varepsilon} p^{l /(2 l-1)}$ количество $x \in Z_{p}^{*}$, представимых в виде $x \equiv b_{1} g^{n_{1}}+\cdots+b_{l} g^{n_{k}}(\bmod p), 1 \leqslant n_{1}, \ldots, n_{l} \leqslant N$, больше, чем $(1-\varepsilon) p$.

ЗАмЕчАниЕ. При условии выполнения обобщенной гипотезы Римана в [2] было показано, что в случае $l=2$ теорема Б верна для $N \gg p^{2 / 3+\varepsilon}$.

2. Доказательство теорем. Пусть $M$ - подмножество $Z_{p}$. Обозначим через $\widehat{\chi}_{M}(r)$ $r$-й коэффициент Фурье характеристической функции $\chi_{M}(x)$ множества $M$, т.е. $\widehat{\chi}_{M}(r)=$ $\sum_{x \in M} e_{p}(r x)$, где $e_{p}(x)=e^{2 \pi i x / p}$. Пусть $l M$ означает сумму $M+\cdots+M l$ раз.

ДокАЗАТЕЛЬСтво теОремЫ 1. Возьмем любое $\varepsilon>0, \varepsilon<1$. Пусть $C_{\varepsilon} \geqslant \varepsilon^{-2 /(2 l-1)}$ и $|R| \geqslant C_{\varepsilon} p^{l /(2 l-1)}$. Тогда $p^{l / 2} /|R|^{(2 l-1) / 2}<\varepsilon$. Рассмотрим сумму $\sigma=\sum_{r \in Z_{p}} \widehat{\chi}_{R}^{l}(r) \widehat{\chi}_{l R}(-r)$. С одной стороны, эта сумма равна 


$$
\sum_{r \in Z_{p}} \widehat{\chi}_{R}^{l}(r) \widehat{\chi}_{l R}(-r)=\sum_{r} \sum_{x_{1} \in R} \cdots \sum_{x_{l} \in R} \sum_{c \in l R} e_{p}\left(x_{1}+\cdots+x_{l}-c\right)=p|R|^{l} .
$$

С другой стороны, $\sigma$ равна $|R|^{l}|l R|+\sum_{r \in Z_{p}^{*}} \widehat{\chi}_{R}^{l}(r) \widehat{\chi}_{l R}(-r)$. Так как для $r \in Z_{p}^{*}$ имеем $\left|\widehat{\chi}_{R}(r)\right|<\sqrt{p}$ (см. [1]), то, применяя неравенство Коши-Буняковского и равенство Парсеваля, получаем

$$
|R|^{l}(p-|l R|) \leqslant p^{(l-1) / 2}\left|\sum_{r \in Z_{p}^{*}} \widehat{\chi}_{R}(r) \widehat{\chi}_{l R}(-r)\right| \leqslant p^{(l+2) / 2}|R|^{1 / 2} .
$$

Следовательно, по выбору $C_{\varepsilon}$ получаем $|l R| \geqslant p\left(1-p^{l / 2} /|R|^{(2 l-1) / 2}\right)>(1-\varepsilon) p$. Теорема доказана.

ДОКАЗАТЕЛЬСтвО тЕОРЕМЫ 2 . Возьмем любое $\varepsilon>0, \varepsilon<1$. Пусть $C_{\varepsilon}^{1} \geqslant \varepsilon^{-2 /(l+1)}, D=$ $\left\{g^{j}: 0 \leqslant j \leqslant C_{\varepsilon}^{1} p^{l /(l+1)}\right\}, E=\{x y: x, y \in D\}$. Пусть также $\lambda_{E}(z)=\#\{x, y \in D: x y \equiv z$ $(\bmod p)\}$, a $\widehat{\lambda}_{E}(r)=\sum_{z \in E} \lambda_{E}(z) e_{p}(r z)$. Заметим, что $\sum_{z} \lambda_{E}(z)=|D|^{2}, \sum_{z} \lambda_{E}^{2}(z) \leqslant|D|^{3}$ и для $r \in Z_{p}^{*}$ имеем $\left|\widehat{\lambda}_{E}(r)\right| \leqslant \sqrt{p}|D|\left(\right.$ см. [1]). Обозначим через $\chi_{A}(x)$ характеристическую функцию множества $A \subseteq Z_{p}^{l-1}$ из $(1)$ с $0 \leqslant n_{1}, \ldots, n_{l} \leqslant 2|D|$, а через $\widehat{\chi}_{A}(\mathbf{r})=\sum_{\mathbf{x} \in A} e_{p}(\mathbf{r} x)-$ ее r-й коэффициент Фурье. Рассмотрим сумму

$$
\begin{gathered}
\sum_{r_{1}, \ldots, r_{m-1}} \widehat{\chi}_{A}\left(-\left(r_{1}, \ldots, r_{m-1}\right)\right) \widehat{\lambda}_{E}\left(r_{1}\right) \widehat{\lambda}_{E}\left(-r_{1}+r_{2}\right) \\
\ldots \widehat{\lambda}_{E}\left(-r_{l-2}+r_{l-1}\right) \widehat{\lambda}_{E}\left(-r_{l-1}\right)=p^{l-1}|D|^{2 l}
\end{gathered}
$$

Член в $(2)$ с $r_{1}=\cdots=r_{l-1}=0$ равен $|A||D|^{2 l}$. Оценим сверху оставшуюся сумму. Разобьем ее на сумму $\sigma_{1}$ по $r_{1} \neq 0$ и на сумму $\sigma_{2}$ по $r_{1}=0$. Пользуясь оценкой для коэффициентов Фурье функции $\lambda_{E}$ и неравенством Коши-Буняковского, получаем

$$
\begin{aligned}
\sigma_{1} \leqslant & \sqrt{p}|D|\left(\sum_{r_{1}, \ldots, r_{l-1}}\left|\widehat{\chi}_{A}\left(-\left(r_{1}, \ldots, r_{l-1}\right)\right)\right|^{2}\right)^{1 / 2} \\
& \times\left(\sum_{r_{1}, \ldots, r_{l-1}}\left|\widehat{\lambda}_{E}\left(-r_{1}+r_{2}\right)\right|^{2} \ldots\left|\widehat{\lambda}_{E}\left(-r_{l-1}\right)\right|^{2}\right)^{1 / 2} \leqslant \sqrt{p}|D| p^{l-1}\left(p|D|^{3}\right)^{(l-1) / 2} .
\end{aligned}
$$

При суммировании по $r_{1}=0$ второй множитель в $(2)$ равен $|D|^{2}$. Как и выше, разбиваем $\sigma_{2}$ на сумму по $r_{2} \neq 0$ и на сумму по $r_{2}=0$ и так далее. Окончательно, из $(2)$ получаем

$$
\begin{aligned}
p^{l-1}|D|^{2 l} & \leqslant|A||D|^{2 l}+\sqrt{p}|D| p^{l-1}\left(p|D|^{3}\right)^{(l-1) / 2}\left(1+\frac{|D|^{2}}{\sqrt{p}|D|^{3 / 2}}+\frac{|D|^{4}}{p|D|^{3}}+\cdots\right) \\
& \leqslant|A||D|^{2 l}+2 \sqrt{p}|D| p^{l-1}\left(p|D|^{3}\right)^{(l-1) / 2}
\end{aligned}
$$

Следовательно, по выбору $C_{\varepsilon}^{1}$, получаем $|A| \geqslant p^{l-1}\left(1-p^{l / 2} /|D|^{(l+1) / 2}\right)>(1-\varepsilon) p^{l-1}$. Итак, существует более $(1-\varepsilon) p^{l-1}$ векторов из $Z_{p}^{l-1}$, принадлежащих $A$. Ровно столько же векторов принадлежат $g A \subseteq A^{*}$, где в определении $A^{*}$ имеем $N \geqslant 3 C_{\varepsilon}^{1} p^{l /(l+1)}$. Теорема доказана.

Доказательство теоремы 3 аналогично доказательствам теорем 1 и 2.

\section{СПИСОК ЛИТЕРАТУРЫ}

[1] S. Konyagin, I. Shparlinski. Character Sums with Exponential Functions and their Applications. Cambridge: Cambridge Univ. Press, 1999. [2] Z. Rudnick, A. Zaharescu // Israel J. Math. 2000. V. 120. P. 271-287. [3] M. Vâjâitu, A. Zaharescu // Int. J. Math. Math. Sci. 2002. V. 29. № 6. Р. 325-331. [4] Г. А. Фрейман. Основания структурной теории сложения множеств. Казань: Казанский гос. пед. ин-т, 1966. 